\title{
The Centennial of the Gibbs-Konovalov Rule for Congruent Points
}

\section{Its Underlying Theoretical Basis and Its Application to Phase Diagram Evaluation}

\author{
By David A. Goodman* \\ John W. Cahn \\ Lawrence H. Bennett \\ Metallurgy Division, Center for Materials Science \\ National Bureau of Standards
}

\begin{abstract}
One hundred years ago, Konovalov developed and tested the rule that at a congruent transformation the phase boundary reaches an extremum. An important equation for the slopes of phase boundaries, derived by Gibbs five years earlier, provides the underlying theoretical basis for the rule, which is still commonly ignored. Applications of the GibbsKonovalov equation to congruent transformations, retrograde solubility, phase boundary inflections, and solid-liquid equilibrium are discussed.
\end{abstract}

Basic thermodynamic principles, such as the phase rule, put constraints on the possible configurations of phase diagrams. One thermodynamic rule developed and tested experimentally by Konovalov ${ }^{1}$ one hundred years ago fixed the slopes of coexistence curves of vaporliquid equilibria of binary solutions. Gibbs gave a more general expression for the slopes of phase boundaries five years earlier. ${ }^{2}$ The specialized form of the GibbsKonovalov equation that we will use appears in a textbook by Schottky, Ulich and Wagner. ${ }^{3}$ This equation is generally applicable and does not depend on any assumptions such as special solution models. It is discussed here to show that a variety of phase diagram features are accounted for by a single equation and to point out its applications, which are often overlooked in research papers, data compilations and textbooks. Because the Gibbs-Konovalov equation is a general thermodynamic relationship, a significant consideration in evaluating phase diagrams is the adherence of phase boundary slopes and curvatures to this thermodynamic requirement.

Frequently, more limited or more awkward equations for special applications are proposed. In the classic textbook by Darken and Gurry, ${ }^{4}$ for example, there is a useful discussion of the slope of the liquidus at a congruent transformation. The equations used, while correct and derivable from the Gibbs-Konovalov equation, contain too many variables giving the sum of the slopes but not the individual slopes. Even as recently as 1980 , Gokcen ${ }^{5}$ felt obliged to use a more limited equation in

\section{Konovalov, Dmitry Petrovich 1856-1929}

Konovalov graduated from the Institute of Mines in St. Petersburg in 1878. A few years later, he became a professor in the Department of Inorganic Chemistry of St. Petersburg University, where he studied under Mendeleev. In 1904 he became Director of the St. Petersburg Institute of Mines and, in 1907, Director of the Russian Department of Mines. He was deputy minister of trade and industry from 1908 until 1915. From 1922 until his death in 1929, Konovalov was president of the Bureau of Weights and Measures in Leningrad and a member of the International Bureau of Weights and Measures. In 1923 he was elected a member of the Soviet Academy of Sciences.

Konovalov's basic works are in the physical chemistry of solutions, kinetics, and catalysis. Konovalov investigated the pressure-temperature-composition relationships for multicomponent vapor-liquid equilibria. In his dissertation (1881, Leipzig) and a later publication in 1884, he established that, compared with the solution, the vapor contains an abundance of that component which, when added to the solution, increases the general vapor pressure of the latter. At the points corresponding to the maximum and minimum of the pressure-composition curve, the vapor has precisely the same composition as the liquid. These laws entered the chemical literature as Konovalov's laws and confirmed the theoretical predictions of Gibbs. This work in the basic thermodynamics of solutions provides a scientific basis for the important industrial process of distillation. 


\section{Goodman et al.}

a discussion of retrograde solubility. For these and other applications, it is just as easy to use the GibbsKonovalov equation.

We propose to sketch out the derivation of the GibbsKonovalov equation for temperature-composition variations and to show its application to various phase diagram features, including congruent transformations, Van't Hoff's equation, phase boundary inflections, retrograde solubility, and freezing ranges in alloys. Finally, we discuss in detail the shape of a coexistence curve for solid-liquid equilibrium.

\section{Derivation of the Gibbs-Konovalov equation}

The GK equation for the slope of the coexistence curve between the phases $\alpha$ and $\beta$ for binary alloys of components $A$ and $B$ at constant pressure is ${ }^{3}$ :

$$
\left(\frac{d T}{d X_{A}^{\alpha}}\right)_{\text {coex }}=\frac{\left(X_{A}^{\alpha}-X_{A}^{\beta}\right) T G_{x x}^{\alpha}}{\Delta H_{F P}^{\beta}}
$$

$X_{A}^{\alpha}$ is the mole fraction of $A$ in the $\alpha$ phase. $G_{x x}^{\alpha}$ is the second compositional derivative of the Gibbs energy of the $\alpha$ phase, $\left(\partial^{2} G^{\alpha} / \partial\left(X_{A}^{\alpha}\right)^{2}\right)_{T} . \Delta H_{F P}^{\beta}$ is the molar enthalpy of first precipitation of an infinitesimal amount of the $\beta$ phase from the $\alpha$ phase. Various equivalent forms of $\Delta H_{F P}^{\beta}$ have been given; Schottky et al. ${ }^{3}$ and Wagner ${ }^{6}$ use Eq 2a, Cahn et al. ${ }^{7}$ use Eq $2 \mathrm{~b}$, and Van der Waals and Kohnstamm ${ }^{8}$ use Eq 2c:

$$
\begin{array}{ll}
\Delta H_{F P}^{\beta}=X_{A}^{\beta}\left(\bar{H}_{A}^{\beta}-\bar{H}_{A}^{\alpha}\right)+X_{B}^{\beta}\left(\bar{H}_{B}^{\beta}-\bar{H}_{B}^{\alpha}\right) & \text { Eq 2a } \\
\Delta H_{F P}^{\beta}=H^{\beta}-\left(X_{A}^{\beta} \bar{H}_{A}^{\alpha}+X_{B}^{\beta} \bar{H}_{B}^{\alpha}\right) & \text { Eq 2b } \\
\Delta H_{F P}^{\beta}=H^{\beta}-H^{\alpha}-\left(X_{A}^{\beta}-X_{A}^{\alpha}\right)\left(\frac{\partial H^{\alpha}}{\partial X_{A}^{\alpha}}\right)_{T} & \text { Eq 2c }
\end{array}
$$

where $H^{\alpha}$ is the molar enthalpy of the $\alpha$ phase, and $\bar{H}_{A}^{\alpha}$ is the partial molal enthalpy of component $A$ in the $\alpha$ phase, evaluated at the equilibrium $X^{\alpha}$. The $H^{\beta}$ and $\bar{H}^{\beta}$ are evaluated at the equilibrium $X^{\beta}$.

The equation for ( $d T / d X_{A}^{\beta}$ ) can be obtained from $\mathrm{Eq} 1$ and 2 by interchanging $\alpha$ and $\beta$.

$$
\left(\frac{d T}{d X_{A}^{\beta}}\right)_{\text {coex }}=\frac{\left(X_{A}^{\beta}-X_{A}^{\alpha}\right) T G_{x x}^{\beta}}{\Delta H_{F P}^{\alpha}}
$$

Note that the magnitudes of $G_{x x}$ and $\Delta H_{F P}$ are different in the two phases.

We sketch the derivation of Eq 1 in order to reveal its scope and limitations. The basic condition of thermodynamic equilibrium for a first order transition is that the chemical potential, $\bar{G}_{A}$, of component $A$ in the $\alpha$ and $\beta$ phases are identically equal along the coexistence curve. The equation for component $A$ (and a similar equation for component $B$ ) is:

$\bar{G}_{A}^{\alpha}-\bar{G}_{A}^{\beta}=0$

Eq 4

Assuming that the derivatives exist and are finite, the total differential of $\mathrm{Eq} 4$ for the variables $T$ and $X$ is:
$\frac{\left(\bar{H}_{A}^{\beta}-\bar{H}_{A}^{\alpha}\right) d T}{T}+\left(\frac{\partial \bar{G}_{A}^{\alpha}}{\partial X_{A}^{\alpha}}\right)_{T} d X_{A}^{\alpha}-\left(\frac{\partial \bar{G}_{A}^{\beta}}{\partial X_{A}^{\beta}}\right)_{T} d X_{A}^{\beta}=0$

Eq 5

Multiplying Eq 5 by $X_{A}^{\alpha}$, and a similar equation for $B$ by $X_{B}^{\alpha}$, adding the resultant equations together, and simplifying with the Gibbs-Duhem relation:

$-X_{A}^{\beta}\left(\frac{\partial \bar{G}_{A}^{\beta}}{\partial X_{A}^{\beta}}\right)_{T}=X_{B}^{\beta}\left(\frac{\partial \bar{G}_{B}^{\beta}}{\partial X_{A}^{\beta}}\right)_{T}$

(and a similar Gibbs-Duhem equation for $B$ ), yields $\mathrm{Eq} 1$, or in terms of activities,

$\left(\frac{d T}{d X_{A}^{\alpha}}\right)_{\text {coex }}=\frac{\left(\frac{X_{A}^{\alpha}-X_{A}^{\beta}}{X_{B}^{\alpha}}\right) R T^{2}\left(\frac{\partial \ln a_{A}^{\alpha}}{\partial X_{A}^{\alpha}}\right)_{r}}{X_{A}^{\beta} \Delta \bar{H}_{A}+X_{B}^{\beta} \Delta \bar{H}_{B}}$

The GK equation, Eq 1 or 7, describes general phase equilibrium in binary systems; it is only limited to first order transitions. Note that the data requirements for application of Eq 1 are the partial molal enthalpies of $A$ and $B$ in the two coexisting phases, i.e., $\Delta \bar{H}_{A}$ and $\Delta \bar{H}_{B}$, and the second compositional derivative of the Gibbs energy of the same phase, $G_{x x}^{\alpha}$.

\section{Applications}

A number of interesting applications follow directly from an examination of the several factors in $\mathrm{Eq} \mathrm{1:}$ congruent transformations when $\left(X_{A}^{\alpha}-X_{A}^{\beta}\right)=0$, retrograde points when the denominator vanishes, inflections near where $\left(\partial^{2} G / \partial X^{2}\right)_{T}$ has a minimum and special considerations in dilute solutions where $\left(\partial^{2} G / \partial X^{2}\right)_{T}$ tends to infinity. Other applications follow from quantitative assessment of the factors.

Congruent Transformations. In the situation of a congruent first order transformation, the compositions of the $\alpha$ and $\beta$ phases are the same. When $\left(\partial^{2} G / \partial X^{2}\right)$ remains finite $\left(G_{x x}\right.$ becomes infinite for a solute in a pure component) and the denominator is not equal to zero, then $(d T / d X)_{\text {coex }}=0$. Thus, the coexistence curves pass through a minimum, maximum, or inflection* with zero slope. Also, when $\left(\partial^{2} G / \partial X^{2}\right)_{T}$ is greater than zero and one branch of a coexistence curve passes through an extremum, then $\left(X_{A}^{\alpha}-X_{A}^{\beta}\right)=0$; the transformation is congruent. Because the slope of the other branch of the coexistence curve is also proportional to $\left(X_{A}^{\alpha}-X_{A}^{\beta}\right)$, the other branch too has zero slope at this point. Thus, when one branch of a coexistence curve reaches an extremum, the other branch of the coexistence curve must touch it and reach an extremum at that point. This was first demonstrated by Konovalov for vapor-liquid equilibrium.

*A congruent point at which the coexistence curve has a horizontal inflection can exist in binary alloys at a point with no degrees of freedom in $T-X-P$ space and thus only at fixed pressure. On one side of this pressure, there will be a pair of congruent points, one a minimum and the other a maximum. With changing pressure, minimum and maximum coalesce and thereafter no congruent point exists. Unless pressure is an explicit variable in the study, it is unlikely that such a feature will ever be part of a binary phase diagram. Congruent inflection points that are not horizontal have been proposed by Goroshchenko, Russ. $J$. Inorg. Chem, , 16(5), p 625 (1971). They are clearly not allowed by the GK equation at any pressure. 
Many phase diagram compilations, textbooks and research articles are in disagreement with these thermodynamic arguments. ${ }^{4}$ Two examples are taken from Hansen ${ }^{9}$ : U-Ni and Th-Zr. Selection of Hansen to illustrate this common error is not meant to imply that these errors are more common in this compilation than others. In fact, almost every compilation of phase diagrams has examples of this error.

Figure 1 gives the uranium-nickel phase diagram. ${ }^{9}$ In this diagram, the liquidus slope is discontinuous at the congruent melting point of $\mathrm{UNi}_{5}$, whereas it is required by Eq 1 to be continuous with a zero slope at the maximum. Note also that the U-rich liquidus of $\mathrm{UNi}_{5}$ is convex while the Ni-rich is concave; we will return to this in the final section entitled "A Detailed Discussion of the Congruent Transformation". 'The solidus comes to a point, but because the $\mathrm{UNi}_{5}$ phase is of narrow stoichiometry, the phase boundary curvature is large. This large curvature causes the solidus to appear pointed. The final section also discusses the conditions under which the coexistence curves can appear pointed for a liquid-solid transformation

Hansen provides two diagrams for the $\mathrm{Zr}$-Th system, one of which (Fig. 2a) shows an incorrect construction of the liquidus. This version has a smooth solidus curve with a zero slope at the minimum, which unfortunately appears displaced from the point of congruency, but even more seriously in error the liquidus slope is discontinuous at this point, in violation of thermodynamic arguments. The second $\mathrm{Zr}$-Th diagram, Fig. $2 \mathrm{~b}$ is consistent with Eq 1. At the congruent melting point, both curves flatten out and reach a minimum.

Van't Hoff's Equation. For infinitely dilute solutions (as $X_{B}^{\alpha}$ approaches 1$),\left(\partial^{2} G^{\alpha} / \partial X^{2}\right)_{T}$ approaches infinity (as $R T / X_{A}^{\alpha}$ ) and Eq 1 becomes indeterminate, but ( $d T / d x$ ) approaches a finite limit. This leads to the Van't Hoff equation:

$\operatorname{limit}_{X_{A}^{\mathrm{a}} \rightarrow 0}\left(\frac{d T}{d X_{A}^{\alpha}}\right)_{\text {coex }}=(1-K) \frac{R T^{2}}{\Delta H_{B}^{\alpha \rightarrow \beta}}$

where $\Delta H_{F P}$ becomes the enthalpy of transformation of pure $B$ for the $\alpha$ to $\beta$ transition: $\Delta H_{F P}=\Delta H_{\beta}^{\alpha \rightarrow \beta}$, and $K$ is the limiting value of the partition coefficient, $x_{A}^{\beta} / x_{A}^{\alpha}$. Note that for an infinitely dilute solution, the difference in the reciprocal slopes of the two branches of the coexistence curves depends only on the temperature and heat of transformation of the major component and is independent of the nature of the solute: $\left(d X_{A}^{\alpha} / d T\right)-$ $\left(d X_{A}^{\beta} / d T\right)=\Delta H_{B}^{\alpha \rightarrow \beta} / R T_{0}^{2}$ where $\mathrm{T}_{0}$ is the transformation temperature of the pure solvent. This equation, which is independent of any solution model, can be used to evaluate the consistency of a series of phase diagrams based on the same component.

Division of Phase Diagram Error. Another point to consider is a common error ${ }^{10}$ presented in many metallurgical, materials, and chemical textbooks. Complex phase diagrams consisting of a series of eutectics between line compounds and their components are often described as being composed of two independent, simple eutectic diagrams. This error leads to diagrams like Fig. 3a. The liquidus curve has a nonzero slope at the congruent transformation point. Only at the terminal solutions (or completely associated or completely or-
Fig. 1 Portion of the U-Ni Phase Diagram (Shown by Hansen ${ }^{9}$ )

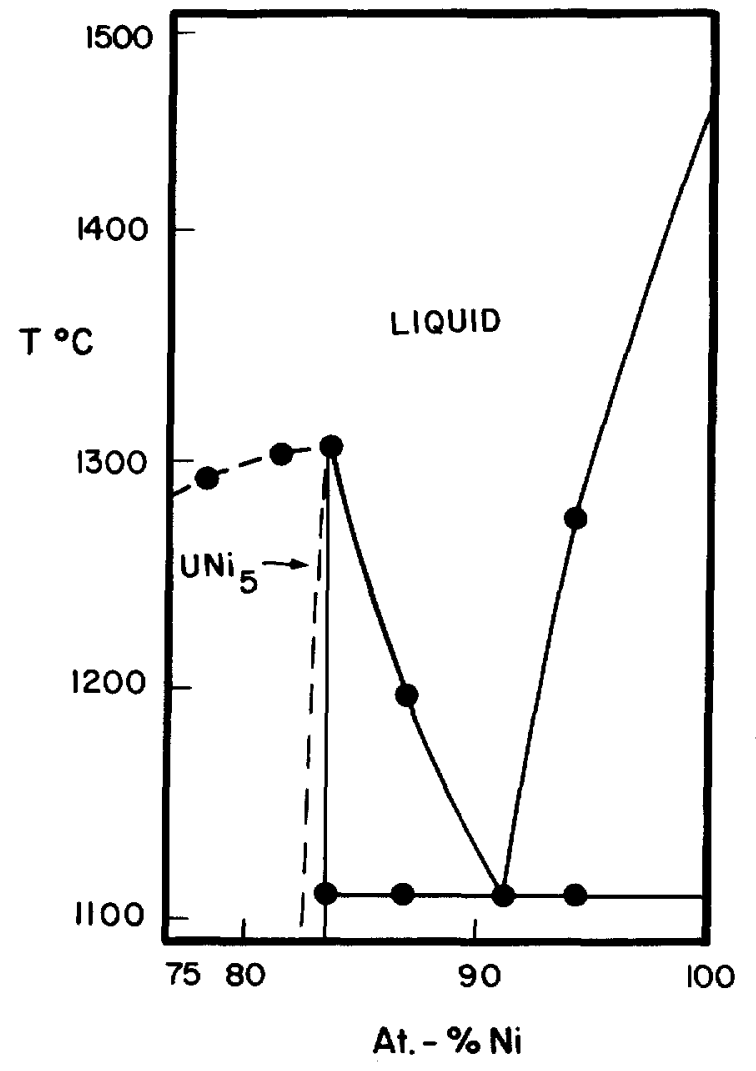

dered phases that act as a pure component) will $\left(\partial^{2} G / \partial X^{2}\right)_{T}$ increase without limit for all phases to give rise to nonzero slopes. The correct diagram is given in Fig. 3b. Even though the solid phase may be a line compound, $G_{x x}$ for the solid does not affect the slope of the liquidus at the congruent point. The liquidus will be smooth unless complete ordering or association occurs in the liquid phase. While it may be a useful pedagogical device to divide and conquer phase diagrams, the correct construction of phase diagrams depends on a unified structure built on a thermodynamic foundation.

The Relationship Between Inflections in Phase Boundaries and Nearby Consolute Critical Points. The GK equation can be used to explain an unusual feature found in several phase diagrams. The Au-Pt (this diagram appears in this issue of the Bulletin), $\mathrm{Ag}-\mathrm{Zr}$, and $\mathrm{Cu}$-Rh systems have an inflection point in the solidus near the critical point of a miscibility gap (the consolute point). Also, the solubility curve of $\mathrm{Ag}$ in $\mathrm{Al}$ has an inflection point near a metastable miscibility gap. ${ }^{11}$ These features are readily explained. At the extremum of a miscibility gap, $\left(\partial^{2} G / \partial X^{2}\right)_{T}=0$. For temperatures and compositions near the miscibility gap, the curvature of the free energy will be nearly zero in that phase. Because ( $d T / d X$ ) is proportional to $\left(\partial^{2} G / \partial X^{2}\right)_{T}$, all phase boundaries of that phase near a miscibility gap will flatten out and have an inflection point. No inflection point is necessary for the other 


\section{Goodman et al.}

Fig. 2a Portion of a Zr-Th Phase Diagram That Violates the Gibbs-Konovalov Rule

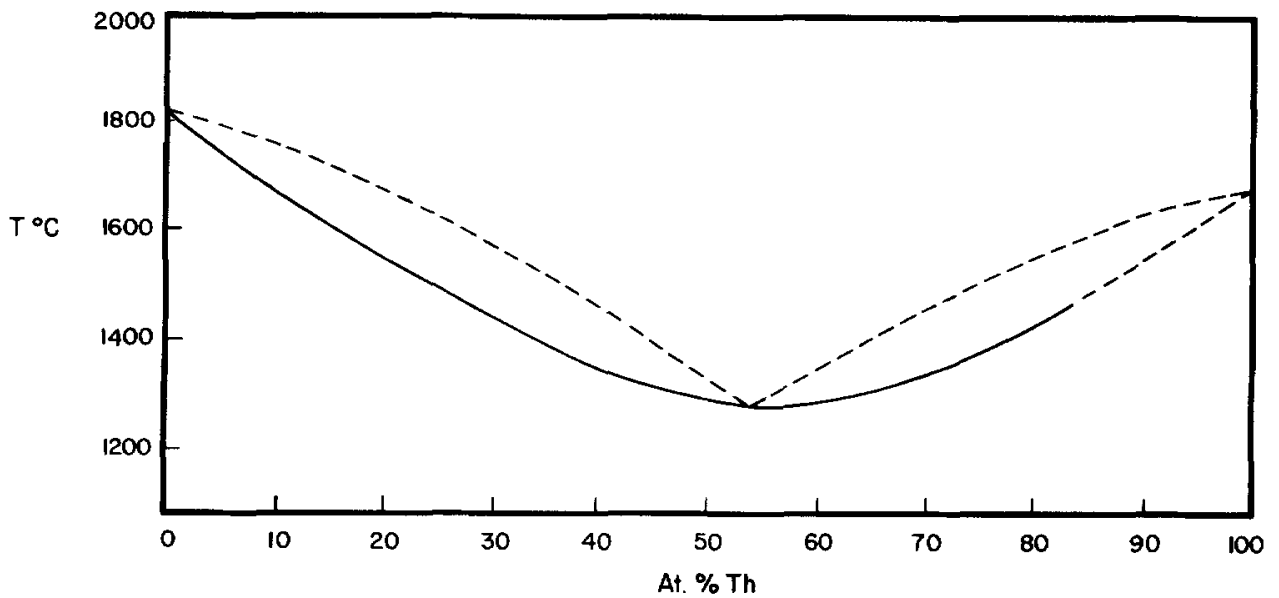

Solid line is the solidus; dashed line is the liquidus. From Fig. 671 in Hansen. ${ }^{9}$

Fig. 2b Portion of a Zr-Th Phase Diagram Consistent with the Gibbs-Konovalov Rule

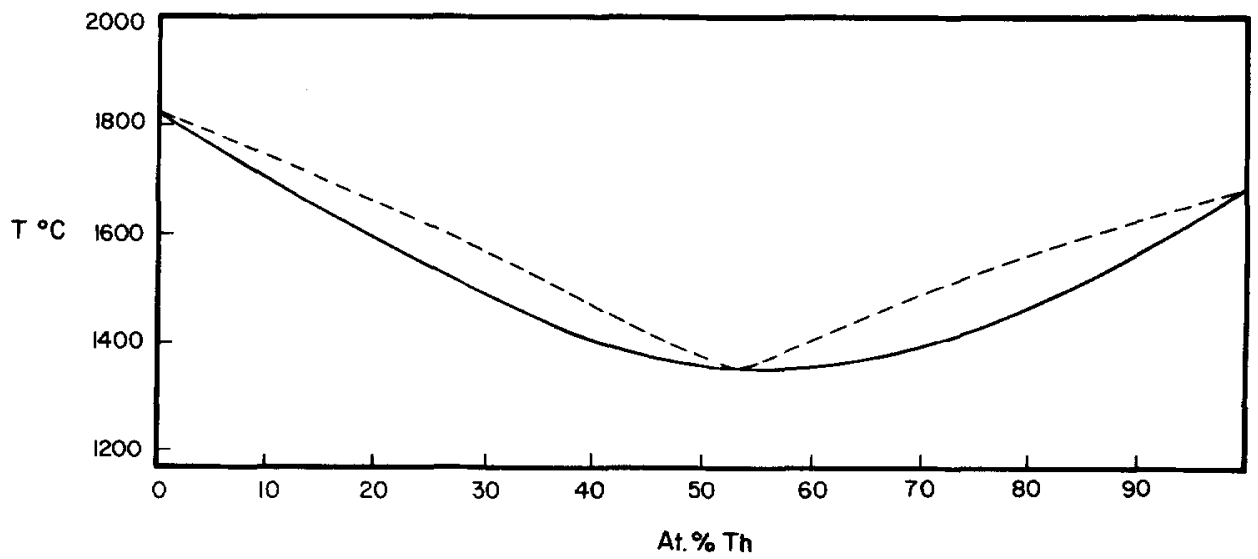

Solid line is the solidus; dashed line is the liquidus. From Fig. 672 in Hansen. ${ }^{9}$

phase boundary, e.g., the liquidus in Au-Pt, because ( $\partial^{2} G / \partial \mathrm{X}^{2}$ ) would only tend to zero if the liquid phase is also about to separate into two liquid phases. Because inflection points can also arise from other factors in the GK equation, the converse, inferring a critical point from an inflection, does not always hold.

Retrograde Solubility. This feature of phase diagrams, which is found in the $\mathrm{Zn}-\mathrm{Cd}, \mathrm{Ag}-\mathrm{Pb}, \mathrm{Cu}-\mathrm{Fe}$, and other phase diagrams, can only octur when the denominator in $\mathrm{Eq} 1$ vanishes. ${ }^{7,12} \mathrm{~A}$ necessary condition is that the quantities $\left(\bar{H}_{A}^{\beta}-\bar{H}_{A}^{\alpha}\right)$ and $\left(\vec{H}_{B}^{\beta}-\bar{H}_{B}^{\alpha}\right)$ both are zeor have opposite signs. In the unlikely event that both $\Delta \bar{H}$ are zero at a given temperature, both coexistence curves will have a retrograde point at the same tem- perature. More generally, a retrograde solubility occurs when the $\Delta \bar{H}$ have opposite signs, so that when one curve is retrograde at a given temperature the other is not. It is apparent that retrograde points have nothing to do with phase diagram features that depend on factors other than $\Delta \bar{H}^{5}$

Freezing Range in Alloys. Wagner ${ }^{13}$ has used Eq 1 to calculate the temperature gap between the liquidus and solidus in the Ag-Au system (see Bull. Alloy Phase Dia., $1(2), \mathrm{p} 45,1980)$. At $X_{\mathrm{Au}}=0.5$, Wagner calculates $\Delta T=1.3 \mathrm{~K}$ compared to $\Delta T=10 \mathrm{~K}$ from early experiments. Later experiments confirm Wagner's prediction; White $^{14}$ reports $\Delta T=1.4 \pm 0.08 \mathrm{~K}$. 


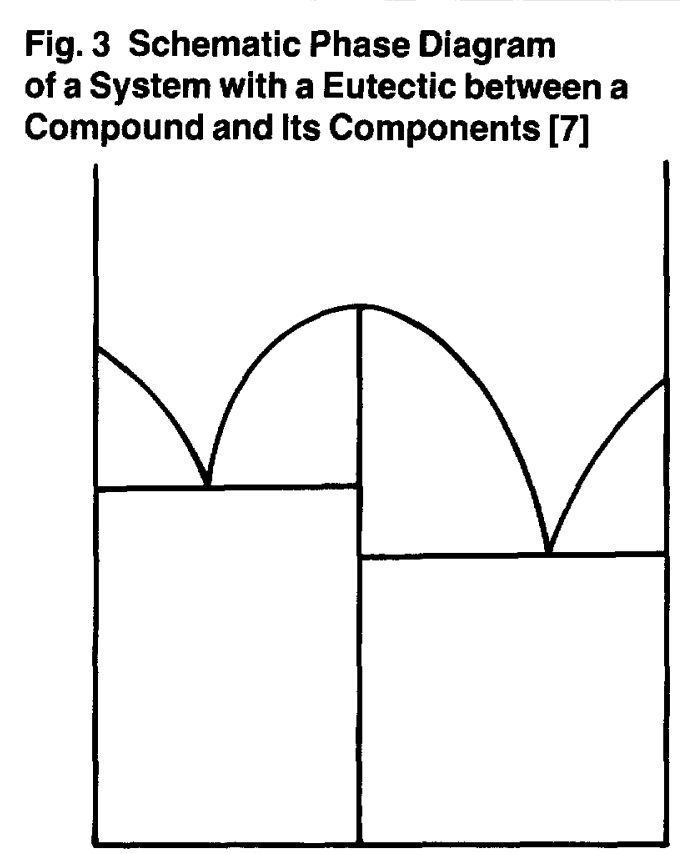

A. Diagram consistent with Gibbs-Konovalov rule.

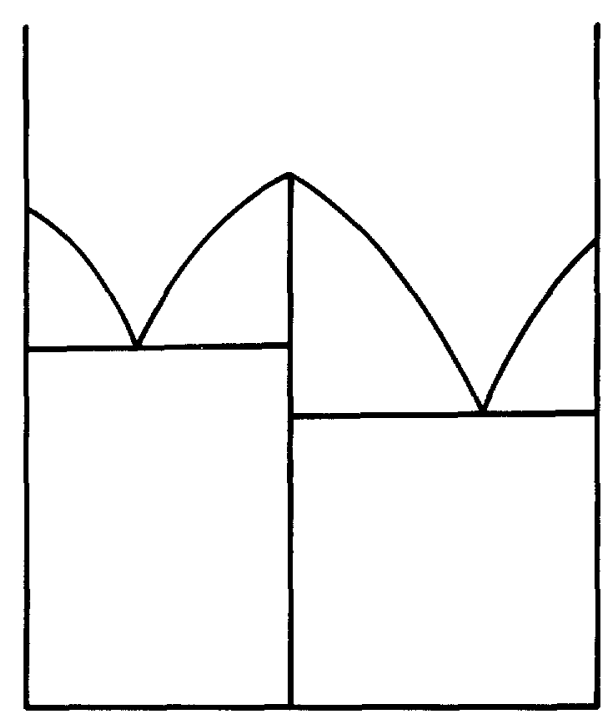

B. Diagram that violates Gibbs-Konovalov rule.

\section{A Detailed Discussion of the Congruent Transformation}

A problem occurs when there are steep coexistence curves associated with narrow composition ranges of intermediate phases. In these instances, it might be difficult for a draftsman to draw a correct curve while using a consistent scale. Although $\mathrm{Eq} 1$ predicts zero slopes at the congruent transformation point $\left(X_{0}, T_{0}\right)$, either coexistence curve can appear pointed if the magnitude of $\left(d^{2} T / d X^{2}\right)$ is large.

The derivative of Eq 1 evaluated at the congruent com- position is found as follows. The derivative of Eq 1 with respect to $X_{A}^{\alpha}$ is ${ }^{15,16}$ :

$$
\begin{aligned}
\left(\frac{d^{2} T}{d\left(X_{A}^{\alpha}\right)^{2}}\right)_{\text {coex }}= & \left(X_{A}^{\alpha}-X_{A}^{\beta}\right) \frac{d}{d X_{A}^{\alpha}}\left(T G_{x x}^{\alpha} / \Delta H_{F P}^{\beta}\right) \\
& +\left(T G_{x x}^{\alpha} / \Delta H_{F P}^{\beta}\right) \frac{d}{d X_{A}^{\alpha}}\left(X_{A}^{\alpha}-X_{A}^{\beta}\right)
\end{aligned}
$$

At $X_{A}^{\alpha}=X_{A}^{\beta}$ :

$\left(\frac{d^{2} T}{d\left(X_{A}^{\alpha}\right)^{2}}\right)_{T_{0}, X_{0}}=\left\{T G_{x x}^{\alpha} / \Delta H^{\alpha \rightarrow \beta}\right\} \quad\left(1-\frac{d X_{A}^{\beta}}{d X_{A}^{\alpha}}\right)$ Eq 10

Taking the ratio of $\mathrm{Eq} 1$ and 2 yields:

$$
\left(\frac{d X_{A}^{\beta}}{d X_{A}^{\alpha}}\right)_{T_{0}, X_{0}}=\frac{G_{x x}^{\alpha}}{G_{x x}^{\beta}}
$$

The curvature of the coexistence curve, $T_{x x}^{\alpha}$, at the congruent point is:

$\left(\frac{d^{2} T}{d\left(X_{A}^{\alpha}\right)^{2}}\right)_{T_{0}, X_{0}}=\frac{T_{0} G_{x x}^{\alpha}}{\Delta H^{\alpha \rightarrow \beta}}\left(1-\frac{G_{x x}^{\alpha}}{G_{x x}^{\beta}}\right)$

For the special situation of $G_{x x}^{\alpha} / G_{x x}^{\beta} \ll 1$, which is likely when the $\beta$ phase is a line compound, Eq 12 becomes independent of $G_{x x}^{\beta}$. This result is given by Wagner ${ }^{6}$ in his $\mathrm{Eq} 4$ to 6 for the integrated form and by Gaye and Lupis ${ }^{16}$ in their Eq 41 for the differential form.

The ratio of the curvature of the $\alpha$ and $\beta$ phase boundaries is

$\left(\frac{T_{x x}^{\alpha}}{T_{x x}^{\beta}}\right)_{T_{0}, x_{0}}=\left(\frac{G_{x x}^{\alpha}}{G_{x x}^{\beta}}\right)^{2}$

$\operatorname{Eq} 13$

Referring to the liquidus and solidus of $\mathrm{UNi}_{5}$ (Fig. 1), because the solidus is convex, Eq 13 demands that the liquidus be convex at the congruent point. Thus, the Ni-rich liquidus must have an inflection if it is to pass through the single data point.

If on a phase diagram the scale of temperature is $\phi$ $(\mathrm{K} / \mathrm{mm})$ and the composition scale is $\Delta$ (mole fraction $/ \mathrm{mm}$ ), then the radii of curvature at the coexistence points are:

$r^{\alpha}=\frac{\phi \Delta H^{\alpha \rightarrow \beta}}{\Delta^{2} T_{0}} / G_{x x}^{\alpha}\left(1-G_{x x}^{\alpha} / G_{x x}^{\beta}\right)$

$\mathrm{Eq} 14 \mathrm{a}$

and

$r^{\beta}=r^{\alpha}\left(\frac{G_{x x}^{\alpha}}{G_{x x}^{\beta}}\right)^{2}$

When $r$ is smaller than the line width $(0.3 \mathrm{~mm})$, the coexistence curve will appear pointed. All the thermodynamic parameters in these equations are readily measurable. It is important to note that the curvature of the coexistence curves at the congruent point depends on the second derivative of the Gibbs energy of both phases. Gaye and Lupis ${ }^{16}$ have discussed the effect of the Gibbs energy curvature on phase boundaries.

While the curvature may be readily calculated for any system in which experimental values of $\Delta H$ and $G$ are available, the magnitude of the factors are easily estimated. Consider the congruent melting of an ordered 


\section{Goodman et al.}

compound. The entropy of melting for a pure metal is given by Richard's rule as $1.1 R$. For an ordered compound, the entropy of disordering must be added, thus $\Delta H_{\text {fus }} \approx 1.5 R T_{\mathrm{m}}$.

The curvatures of the free energies are now estimated. For an ideal solution ${ }^{17}$ :

$G_{x x}^{\text {ideal }}=R T / X_{A} X_{B}$

For a regular solution:

$G_{x x}^{R S}=G_{X X}^{\text {ideal }}-8 \Delta H_{f}$ Eq 16

where $\Delta H_{f}$ is the enthalpy of formation of the phase at $X=0.5$, relative to the pure components in the same phase.

For a solid phase of limited stoichiometry or associated liquids, simple models such as the regular solution model are not expected to accurately describe the free energy as a function of composition. The Wagner and Schottky model of substitutional disorder can be used to calculate $\left(\partial^{2} G / \partial X^{2}\right)_{T}$ for an ordered solid. This model may not give quantitative fits to the data for large deviations from stoichiometry, but it is useful as a starting point for estimating the shape of coexistence curves. Wagner and Schottky's ${ }^{18}$ model for an ordered $A B$ compound with substitutional disorder can be used to derive Eq 17.

$G_{x x}^{W S}\left(X_{0}\right)=R T / 4 X_{0} \alpha=\frac{R T}{2 X_{0}} \exp \left(-2 \Delta H_{f} / R T\right)$

$\operatorname{Eq} 17$

where $\alpha$ is the intrinsic defect concentration at the stoichiometric composition. For a regular associated solution Jordan ${ }^{19}$ has given:

$G_{x x}^{R A S}=G_{x x}^{R S}+\frac{2 R T X_{A B}}{X_{1} X_{2}\left(1-X_{A B}\right)}$

where $X_{A B}$ is the mole fraction of the associated complex. Eq 18 can be used to calculate the effect of association on the coexistence curve.

The calculated liquidus is easily plotted as a smooth curve on a conveniently sized phase diagram. For a $6^{\prime \prime} \times 4^{\prime \prime}$ phase diagram covering $1500 \mathrm{~K}$ and the entire composition range, $\phi=10 / \mathrm{mm}$ and $\Delta=0.01 / \mathrm{mm}$. With $T=1000 \mathrm{~K}$ and $X_{0}=0.5, r$ (liquidus) is typically $40 \mathrm{~mm}$, for an ideal liquid. For a regular solution, even with large negative deviations from ideality, the liquidus is broad. For instance, when $\Delta H_{f}=-10,000$ cal / g.at and the liquid is in equilibrium with a solid of constant composition, the radius of curvature is $4 \mathrm{~mm}$. The liquidus of associated liquids have been calculated by Sommer, Eschenweck, and Predel. ${ }^{20}$ They find that the liquidus is not pointed even for a degree of association as high as 0.94 .

The solidus for highly stable or highly ordered phases can appear sharp. Using Eq 14, 16 and 17, we calculate $r$ (solidus) $=7 \mathrm{~mm}$ for $\Delta H_{f}=-2000 \mathrm{cal} / \mathrm{g} \cdot \mathrm{at}$ and $r$ (solidus) $=0.14 \mathrm{~mm}$ for $\Delta H_{f}=-5000 \mathrm{cal} / \mathrm{g} \cdot \mathrm{at}$. Other models of defect equilibria will give different quantitative results. The qualitative result that the solidus curve will be broad for a weakly interacting system but becomes needle sharp for strongly interacting systems should be generally valid. Thus, even though thermodynamics requires a horizontal slope at the melting maximum, the solidus can appear pointed.

In conclusion, both phase boundaries of most congruent transformations will be rounded. The solidus can appear pointed for strongly interacting systems, while the liquidus will be smooth for all but the most strongly interacting components. In the absence of thermodynamic data, one can estimate that the solidus will appear pointed for phases of very limited nonstoichiometry, but the condition of Eq 13 or 14 should be checked when data are available. Phases of wide stoichiometry will have a smooth coexistence curve like the liquidus.

\section{Summary}

The Gibbs-Konovalov equation is presented and applied to various phase diagram features. This equation is not limited by special assumptions; it can be used as the basis for a general discussion of phase boundaries. The Gibbs-Konovalov equation should be used for phase diagram evaluations.

\section{References}

1. Konovalov, D., Ann. Phys., 14, p 34 (1881).

2. Gibbs, J. W., Trans. Conn. Acad., 3, p 108 (1876), cited in The Collected Works of J. Willard Gibbs, Vol. 1, p 99, Yale University Press, New Haven (1948).

3. Schottky, W., Ulich, H. and Wagner, C., Thermodynamik, Berlin, (1929).

4. Darken, L.S. and Gurry, R.W., Physical Chemistry of Metals, McGraw-Hill, NY (1953).

5. Gokcen, N. A., Scripta Met., 14, p 1185 (1980).

6. Wagner, C., Thermodynamics of Alloys, Addison Wesley (1952).

7. Cahn, J.W., Coriell, S. R. and Boettinger, W. J., in Laser and Electron Beam Processing of Metals, White and Peercy, Editors, Academic Press (1979).

8. Van Der Waals, J. D. and Kohnstamm, Ph., Lehrbuch der Thermodynamik, Part 2, p 109, J. A. Barth, Leipzig (1912).

9. Hansen, M., Constitution of Binary Alloys, McGraw-Hill, NY (1958).

10. Berndt, A. F., J. Chem. Ed., 46, p 594 (1969).

11. Williams, R.O. and Easton, D.S., Scripta Met., 8, p 27 (1974); see also comment by R. O. Williams in Bull. Alloy Phase Dia., 1 (2), p 23 (1980).

12. Meijering, J.L., Philips Res. Rep., 3, p 281 (1948).

13. Wagner, C., Acta Met., 2, p 242 (1954).

14. White, J. L., Trans. AIME, 215 , p 178 (1959).

15. Prigogine, I. and Defay, R., Chemical Thermodynamics, Longmans, London (1958).

16. Gaye, H. and Lupis, C. H.P., Met. Trans., 6A, p 1049 (1975).

17. Gokcen, N.A., Thermodynamics, Techscience (1975).

18. Wagner, C. and Schottky, W., Zeit. Phys. Chem., B11, p 163 (1930).

19. Jordan, A.S., Met. Trans, 1, p 239 (1970).

20. Sommer, F., Eschenweck, D. and Predel, B., Ber. Bunsen. Phys. Chem., 82, p 790 (1978). 